\title{
"It ain't what you do, it's the way that you do it": does obesity affect perceptual motor control ability of adults on the speed and accuracy of a discrete aiming task?
}

\author{
David Gaul $^{1}$ [ $\cdot$ Laure Fernandez ${ }^{2} \cdot$ Johann Issartel $^{3}$
}

Received: 27 April 2017 / Accepted: 4 July 2018

(c) Springer-Verlag GmbH Germany, part of Springer Nature 2018

\begin{abstract}
The ability to control speed and accuracy of goal directed aiming tasks underpins many activities of daily living. Recent evidence has begun to suggest that obesity can affect the control of movement. This study evaluated perceptual motor control of 183 normal weight, overweight, and obese participants using a discrete Fitts' task on a digital tablet. In addition, we manipulated tablet orientation to determine whether tablet orientation influences task difficulty with the view to increase the task's constraints. Our study found that the traditional relationship between target distance and target width hold true for each of the three weight groups in both tablet orientations. Interestingly, no significant differences were found for movement time between the groups, while movement kinematics differed between weight groups. Obese participants demonstrated significantly higher peak acceleration values in the horizontal tablet orientation when compared to their normal weight and overweight counterparts. Further to this, obese participants made significantly more errors than normal weight and overweight groups. These findings suggest that obese individuals have altered control strategies compared to their normal weight peers.
\end{abstract}

Keywords Perceptual-Motor $\cdot$ Fitts' Task $\cdot$ Speed $\cdot$ Accuracy $\cdot$ Obesity

\section{Introduction}

Obesity is a major public health concern which is linked with increased risk of cardiovascular disease, stroke, cancer, type 2 diabetes, hypertension, depression, and obstructive sleep apnea (Lee et al. 2012). Overweight and obesity levels have reached epidemic levels. According to World Health Organization figures from 2014, 39\% of men and $40 \%$ of women aged $18+$ were overweight [body mass index (BMI) $\left.>25 \mathrm{~kg} \mathrm{~m}^{-2}\right]$ and $11 \%$ of men and $15 \%$ of women being obese (BMI $>30 \mathrm{~kg} \mathrm{~m}^{-2}$ ) (World Health Organization 2014). This equates to almost 2.5 billion adults being overweight or obese worldwide. Traditionally, obesity has been "considered a problem of the belly rather than of the brain"

David Gaul

david.gaul@itb.ie

1 School of Business, Institute of Technology Blanchardstown, Dublin 15, Ireland

2 Aix-Marseille Université, CNRS, ISM, Marseille, France

3 School of Health and Human Performance, Dublin City University, Dublin 9, Ireland
(Knecht et al. 2008). More recently, however, there has been mounting evidence suggesting a relationship between obesity and cognitive function (Benito-Leon et al. 2013; Miller and Spencer 2014; Prickett et al. 2014; Bove et al. 2016). These studies have found differences in a variety of facets of cognitive function such as visuospatial skill, memory, sensory integration and attention, mathematical ability, and motor skill throughout the lifespan from children to elderly adults (Prickett et al. 2014; Wan et al. 2014; Liang et al. 2014; Wang et al. 2016; Scarpina et al. 2016). A recent study by Gaul et al. (2016) suggested that obesity affects the sensory integration process in a visual-motor coordination task. This study found that morbidly obese adults demonstrated significantly poorer performance during a visual-motor synchronisation task when compared to a healthy weight control group (Gaul et al. 2016). A worrying trend is that a number of longitudinal studies have found associations between midlife obesity and risk for poor neurocognitive and cognitive function (Cournot et al. 2006; Fitzpatrick et al. 2009; Gunstad et al. 2010; Gustafson 2008; Kivipelto et al. 2005; Whitmer et al. 2005). This has led to body composition being considered as a risk factor for the future development of neurodegenerative diseases such as Alzheimer's 
disease and dementia (Kivipelto et al. 2005; Whitmer et al. 2005; Beydoun et al. 2008; Crichton et al. 2012).

The greatest evidence seems to support the argument for obesity altering typical cognitive processes exists in terms of executive function. Executive function is generally defined as the "higher level" or "meta-cognitive" function that manages other more basic cognitive functions in relation with goal-directed behaviour (Etnier and Chang 2009). As such, executive function is frequently considered as consisting of activities such as the planning, coordination, initiation and stopping of behaviours, and the processing of information related to them (Kramer et al. 1994; Alvarez and Emory 2006). Executive function is, therefore, a vital component of the successful completion of motor tasks such as reaching or grasping. These actions require the ability to appropriately coordinate the speed and accuracy of an individual's movement. This trade-off between movement speed and accuracy is known as 'Fitts' law', which is defined by a linear increase of movement time with the increased difficulty of an aiming task (Fitts 1954; see Meyer et al. 1988; Plamondon and Alimi 1997, for reviews). As such, difficulties with executive function can impair the effective performance of many activities of daily living such as brushing one's hair, feeding oneself or picking up items (Kirby et al. 2011). As obesity is already known to negatively impact an individual's quality of life and influence performance of activities of daily living (ADL) as a result of the mechanical consequence of excess weight, any such motor control difficulties could increase the difficulty of everyday tasks (Rosmond and Bjorntorp 2000). The authors sought to examine whether individuals with different BMI categories (normal weight, overweight, and obese) demonstrated differences in terms of speed and accuracy as well as on the kinematics organization of movement during a discrete version of the Fitts' task on a digital tablet. A secondary addressed question was to determine whether manipulation of tablet orientation altered task difficulty and subsequently highlighted any between-group differences that might exist due to altered BMI.

\section{Methods}

\section{Participants}

A total number of 183 (see Table 1) adult participants partook in this study as part of an interactive exhibition at a science gallery. All participants had their height, weight, and body fat measured and BMI calculated prior to participation. This data was used to divide participants into groups based on their BMI $\left(\mathrm{kg} \mathrm{m}^{-2}\right)$ according to the criteria set by the World Health Organization (WHO 2000). Participants were screened in advance with a questionnaire. Participants were excluded if they failed to complete all trials in the testing session. Informed consent was obtained from all individual participants included in the study.

\section{Apparatus and task}

Participants were seated comfortably at a table, facing a graphics tablet (Wacom Ultra Pad A3) placed in both horizontally and vertical positions mounted on a custom rotating stand on the table in front of them. Left-right motion of a hand-held stylus displaced a cursor on the tablet screen via ICE software developed by Marseille University Lab (Human Movement Sciences Institute). The task was to move the cursor, represented by a red vertical line spanning the full height of the tablet, between two targets depicted on the screen as fast and as accurately as possible (i.e., Fitts' task). The target was a rectangle of a given width at a given distance [depending on the index of difficulty (ID)] with a height corresponding to the height of the screen. Movement was recorded along both horizontal and vertical axis; analysis focused solely on movement along the $X$-axis. The position of the stylus on the graphics tablet was sampled at a frequency of $150 \mathrm{~Hz}$.

\section{Recordings and procedure}

A session consisted of 64 discrete aiming movements from one target to the other in 2 different orientations ( 32 horizontal and 32 vertical). There were four separate experimental conditions made up of a combination of two different target
Table 1 Descriptive statistics showing number, gender, mean age, weight, height, and BMI for participants divided by BMI category

\begin{tabular}{lllll}
\hline & Normal weight & Overweight & Obese & Total \\
\hline$N(\%$ of total $)$ & $107(58.5 \%)$ & $58(31.7 \%)$ & $18(9.8 \%)$ & $183(100 \%)$ \\
Male/female & $37 / 70$ & $33 / 25$ & $7 / 11$ & $77 / 106$ \\
Age \pm SD $($ years $)$ & $34.18 \pm 14.09$ & $38.97 \pm 14.42$ & $41.72 \pm 16.55$ & $36.44 \pm 14.63$ \\
Weight \pm SD $(\mathrm{kg})$ & $63.34 \pm 8.17$ & $79.90 \pm 10.20$ & $100.73 \pm 16.81$ & $72.26 \pm 15.60$ \\
Height \pm SD $(\mathrm{cm})$ & $170.56 \pm 8.52$ & $172.36 \pm 10.12$ & $170.94 \pm 9.84$ & $171.17 \pm 9.17$ \\
BMI \pm SD $\left(\mathrm{kg} \mathrm{m}^{-2}\right)$ & $21.72 \pm 1.77$ & $26.79 \pm 1.36$ & $34.34 \pm 4.00$ & $24.57 \pm 4.44$ \\
\hline
\end{tabular}


widths (small and large) and two distances (close and far). Small and large conditions corresponded, respectively, to 0.8 and $3 \mathrm{~cm}$ targets' width. Close and far corresponded, respectively, to 15 and $40 \mathrm{~cm}$ distances between centres of the targets. This led to participants performing at four levels of task difficulty: $\mathrm{ID}=3.32,4.73,5.23$, and 6.64 with $\mathrm{ID}=\log _{2}$ $(2 D / W)$ (Fitts 1954). During the experiment, the participants carried out the 4 blocks of 8 trials ( 4 conditions $\times 2$ repetitions) in both horizontal and vertical orientations. As such, the experiment consisted of one testing session of the following design: 2 tablet orientations $\times 4$ blocks $\times 4$ conditions $\times 2$ repetitions of each condition. The order of trials was randomized across all blocks and the order of which orientation was displayed first was counterbalanced across all participants to remove any order effects. Errors were defined as an overshoot, i.e., movement beyond the external edge of the target outside area. In an event of an undershoot, the trial would continue until the cursor reached the target. A familiarization phase was included at the beginning of each block of trials with a different tablet orientation. This phase included participants being presented with one trial for each of the 4 conditions while receiving verbal instructions from the experiment moderator and on screen visual instruction. The first trial for each condition in each block in addition to all familiarization trials is not analysed to avoid transient behaviour in the analysis.

\section{Data analysis}

The position time series were filtered with a dual-pass, second-order Butterworth filter, using an $8 \mathrm{~Hz}$ cut-off frequency. Velocity and acceleration were subsequently derived using a three-point central difference technique. The analysis focused on movement time (MT, in s), peak acceleration $\left(\mathrm{PA}\right.$, in $\mathrm{m} \mathrm{s}^{-2}$ ), peak velocity $\left(\mathrm{PV}\right.$, in $\left.\mathrm{m} \mathrm{s}^{-1}\right)$, percentage of acceleration time (in \%), and percentage of overshoot (in
$\%)$. The first two trials and last trial for each condition were removed from the analysis to eliminate any learning effects. For each session, measures were averaged across the remaining five trials for each of the four conditions. For each trial, movement time (MT) was defined as the time taken from movement initiation (when 5\% of PV was reached) to entry of the opposite target (Missenard and Fernandez 2011). Percentage overshoot was calculated by determining the number of trials that the participant moved beyond the external edge of the target before coming back into the target area and dividing it by total number of trials.

\section{Statistical analysis}

Repeated-measures ANOVAs were performed between BMI categories (obese, overweight, and normal weight), orientation (vertical and horizontal), target width (small and large), and target distance (close and far) as factors. Sphericity was assessed for each dependent variable and the Greenhouse-Geisser's correction was applied when sphericity was not met. Post hoc analysis using Bonferroni's correction was used to detail significant effects. Statistical significance was set $p<0.05$.

\section{Results}

\section{Movement time (MT, in s)}

There was a significant interaction effect found between tablet orientation and target width, $F(1,180)=5.51, p<0.02$, $\eta_{\mathrm{p}}^{2}=0.014$. Post hoc tests revealed that participants' movement time was significantly greater for vertical orientation $(M=0.83, \mathrm{SE}=0.04,95 \% \mathrm{CI}[0.76,0.91])$ compared to a horizontal orientation $(M=0.73, \mathrm{SE}=0.04,95 \%$ CI $[0.67$,
Fig. 1 Movement times for all four conditions divided by horizontal and vertical tablet orientations when the target width was large (a) and small (b)
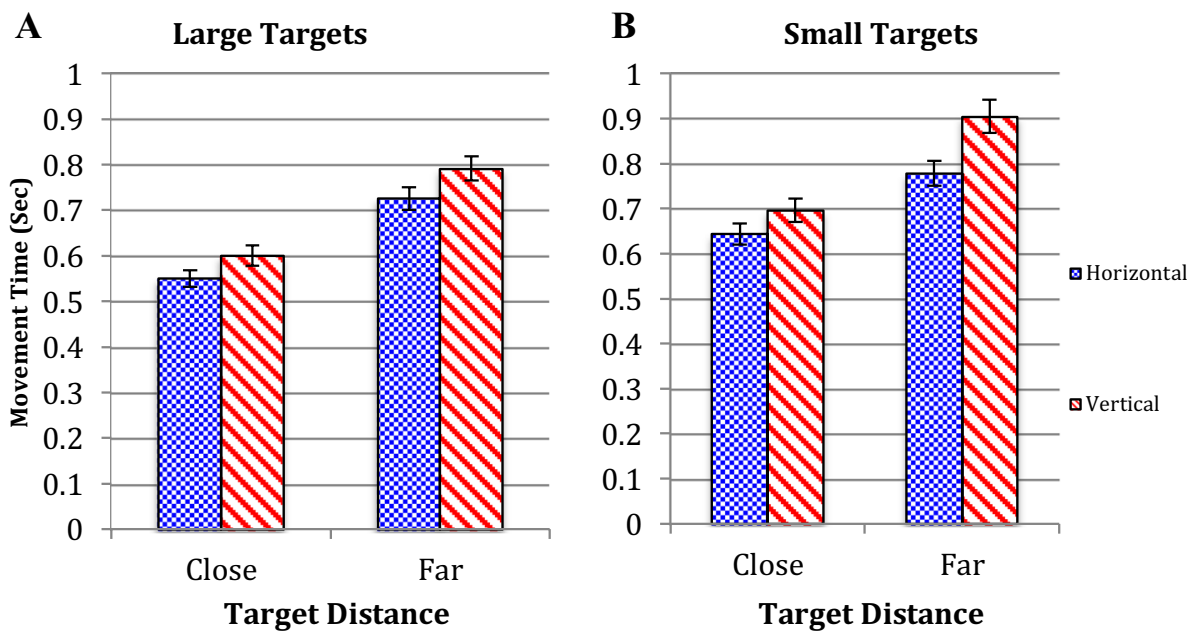
0.79]) when the target was small $(\Delta=13.7 \%)$. Post hoc tests also revealed significantly greater movement time for vertical orientation $(M=0.72, \mathrm{SE}=0.03,95 \% \mathrm{CI}[0.66,0.78])$ compared to horizontal orientation $(M=0.66, \mathrm{SE}=0.03$, $95 \%$ CI $[0.61,0.72])$ when the target was large $(\Delta=9.1 \%)$ (Fig. 1). There was also a significant main effect found for target distance $F(1,180)=143.68, p<0.01, \eta_{\mathrm{p}}^{2}=0.25$, with all participants having greater movement times when the target was farther away $(M=0.83, \mathrm{SE}=0.03,95 \% \mathrm{CI}[0.76,0.89])$ compared to the closer target distance $(M=0.65, \mathrm{SE}=0.03$, $95 \%$ CI $[0.60,0.70])$. There were no significant interaction effects found for BMI category and tablet orientation, width, or distance or any main effect found for BMI category, $F(2,180)=1.08 p>0.05$.

\section{Peak acceleration (PA, in $\mathrm{m} \mathrm{s}^{-2}$ )}

There was a significant interaction effect found between tablet orientation and BMI category, $F(2,180)=3.63, p<0.05$, $\eta_{\mathrm{p}}^{2}=0.04$. Post hoc tests revealed that the obese individuals had significantly higher values for horizontal conditions $(M=624.73, \mathrm{SE}=90.76,95 \%$ CI $[445.63,803.82]) \mathrm{com}-$ pared to vertical conditions $(M=451.19, \mathrm{SE}=88.87,95 \%$ CI $[275.83,626.55])(\Delta=38.5 \%)$ when compared to their normal weight $(M=499.57, \mathrm{SE}=37.2395 \%$ CI [426.12, 573.03] and $M=530.12, \mathrm{SE}=36.45,95 \%$ CI [458.2, 602.04] $)(\Delta=-5.8 \%)$ and overweight $(M=499.35$, $\mathrm{SE}=50.5695 \% \mathrm{CI}[399.58,599.13]$ and $M=486.89$, $\mathrm{SE}=49.5195 \%$ CI $[389.2,584.58])$ peers $(\Delta=2.6 \%)$, respectively (Fig. 2).

There were also significant interaction effects found between distance and orientation, $F(1,180)=5.92, p<0.05$, $\eta_{\mathrm{p}}^{2}=0.03$. Following post hoc analysis, it was revealed that there were significantly higher values for peak acceleration in horizontal orientation $(M=696.43, \mathrm{SE}=55.6,95 \% \mathrm{CI}$ [586.73, 806.14]) compared to vertical orientation $(M=603.05, \mathrm{SE}=53.07,95 \% \mathrm{CI}[498.32,707.78])$ when the targets were far away $(\Delta=13.4 \%)$. There was also a significant main effect found for target width, $F(1,180)=4.33$, $p<0.05, \eta_{\mathrm{p}}^{2}=0.02$, with participants demonstrating greater peak acceleration values for large targets $(M=522.21$, $\mathrm{SE}=34.69,95 \% \mathrm{CI}[453.76,590.66])$ compared to smaller targets $(M=508.41, \mathrm{SE}=32.58,95 \% \mathrm{CI}[444.12,572.71])$.

\section{Peak velocity $\left(P V\right.$, in $\left.\mathrm{m} \mathrm{s}^{-1}\right)$}

There was a significant interaction effect found between tablet orientation and target distance, $F(1,180)=21.98$, $p<0.01, \eta_{\mathrm{p}}^{2}=0.11$. Post hoc tests revealed significantly higher peak velocities for the horizontal orientation $(M=120.05, \mathrm{SE}=4.58,95 \% \mathrm{CI}[111.02,129.08]$ compared to vertical orientation $(M=108.52, \mathrm{SE}=4.41,95 \% \mathrm{CI}$ [99.82, 117.22]) when the target was farther away (Fig. 3). There was also a significant main effect found for target width, $F(1,180)=21.49, p<0.01, \eta_{\mathrm{p}}^{2}=0.11$, with higher peak velocities for large targets $(M=86.37, \mathrm{SE}=2.96,95 \% \mathrm{CI}$ [80.53, 92.21]) compared to small targets $(M=84.35$, $\mathrm{SE}=2.87,95 \% \mathrm{CI}[78.70,90.01])$. There was no significant interaction effect found for BMI category and tablet orientation, width or distance, or any main effect for BMI category on its own, $F(2,180)=0.27, p>0.05$.
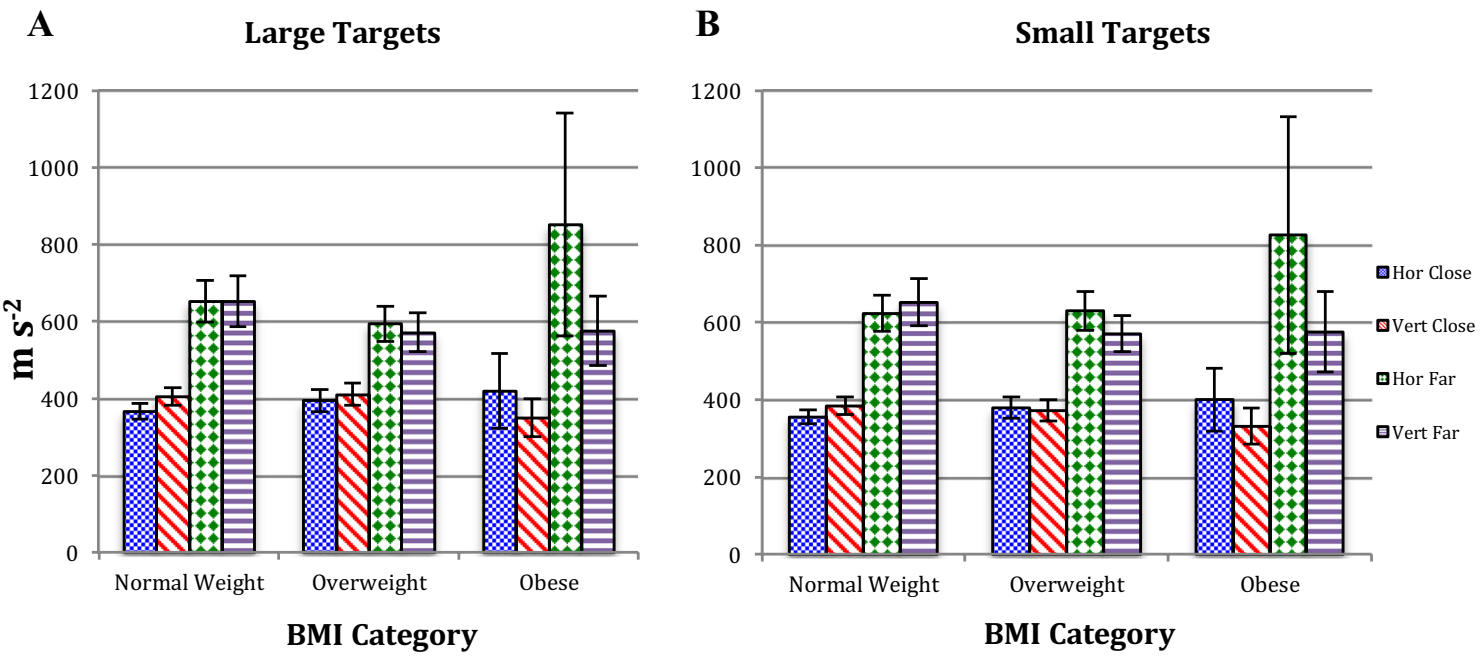

Fig. 2 Peak acceleration values shown for normal weight, overweight, and obese BMI categories when the target width was large (a) and small (b) in both horizontal (Hor close and Hor far) and vertical (Vert close and Vert far) conditions 
Fig. 3 Peak velocity (PV) values shown for both horizontal and vertical orientations when the targets were close and far when the target width was large (a) and small (b) in both horizontal and vertical conditions
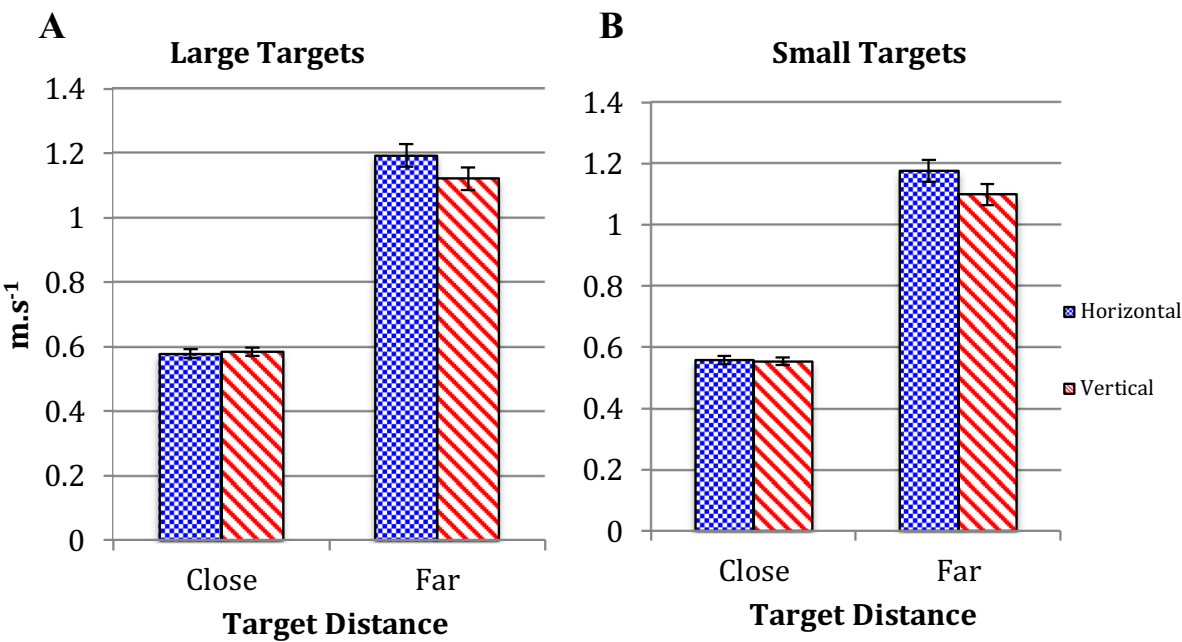

Target Distance

\section{Percentage of acceleration time (in \%)}

There was significant interaction between BMI category and target distance $F(2,180)=3.85, p<0.05, \eta_{\mathrm{p}}^{2}=0.04$. Post hoc tests showed that both normal weight and overweight participants spent a significantly greater percentage of time accelerating in the conditions when the targets were farther away $(M=43.50 \%, \mathrm{SE}=0.57,95 \%$ CI $[42.37,44.63]$ and $M=45.38 \%, \mathrm{SE}=0.7895 \% \mathrm{CI}[43.84,46.92])$ compared to when they were close $(M=42.20 \%, \mathrm{SE}=0.6695 \% \mathrm{CI}$ $[40.89,43.50]$ and $M=42.11 \%, \mathrm{SE}=0.90,95 \%$ CI [40.34, 43.88]) ( $\Delta=1.3 \%$ and 3.3\%) (Fig. 4). There was also a main effect found for width, $F(1,180)=86.21, p<0.01, \eta_{\mathrm{p}}^{2}=0.3$, with participants spending significantly great percentage of time accelerating when the target were large $(M=44.26 \%$, $\mathrm{SE}=0.59,95 \% \mathrm{CI}[43.09,45.42])$ compared to when the target was small $(M=41.59 \%, \mathrm{SE}=0.59,95 \%$ CI $[40.43$,
42.76]). There was no significant interaction effect found between BMI category and orientation. However, there was a trend for obese participants, $F(2,180)=2.68, p=0.06$, to spend less time in the acceleration phase, while the tablet was in the vertical orientation $(M=40.84 \%, \mathrm{SE}=1.40,95 \%$ CI $[38.10,43.58])$ compared to the horizontal position $(M=43.52 \%, \mathrm{SE}=1.75,95 \% \mathrm{CI}[40.06,46.98])(\Delta=2.7 \%)$. This is in contrast to overweight $(M=44.27 \%$ and $43.23 \%)$ and normal weight $(M=42.82 \%$ and $M=42.87 \%)$ participants $(\Delta=-2.3 \%$ and $\Delta=0.1 \%$, respectively).

\section{Overshoot rate (in \%)}

There was a significant interaction effect found between BMI category and target distance, $F(2,180)=3.67, p<0.05$, $\eta_{\mathrm{p}}^{2}=0.04$. Post hoc analysis revealed obese $(M=13.33$, $\mathrm{SE}=1.92,95 \% \mathrm{CI}[9.54,17.13])$ participants to overshoot
Fig. 4 Percentage of time spent in acceleration phase of movement for all four conditions divided by horizontal and vertical tablet orientations shown for BMI categories (normal weight, overweight, and obese) when the target width was large (a) and small (b)
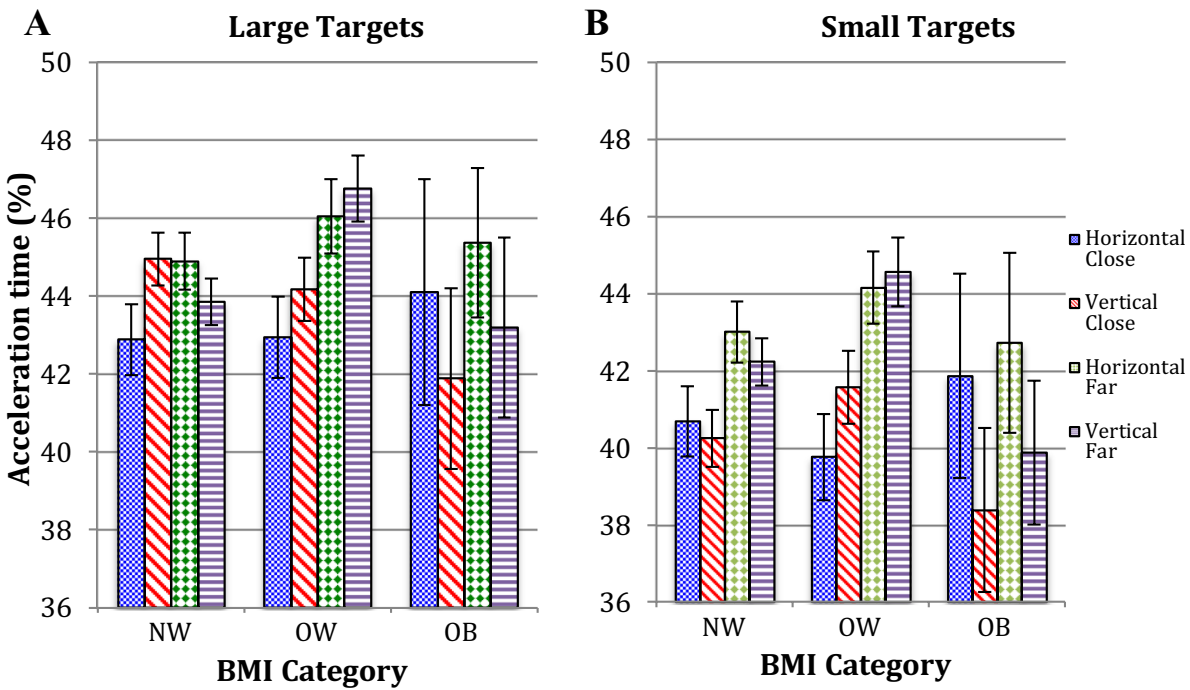
the target a significantly higher number of times compared to normal weight $(M=6.45, \mathrm{SE}=0.79,95 \% \mathrm{CI}[4.89,8.00])$ and overweight $(M=6.21, \mathrm{SE}=1.07,95 \% \mathrm{CI}[4.01,8.32])$ peers only when the target distance was farther. There was a significant main effect found for target width, $F(1,180)=178.02, p<0.01, \eta_{\mathrm{p}}^{2}=0.50$. Participants made more errors when the target was small $(M=16.68, \mathrm{SE}=1.21$ 95\% CI $[14.30,19.06])$ compared to when the targets were large $(M=1.74, \mathrm{SE}=0.34,95 \% \mathrm{CI}[1.07,2.42])$.

\section{Discussion}

The results obtained in this study found that despite exhibiting comparable movement times (Fig. 1) to their normal weight and overweight counterparts, obese participants demonstrated altered control of movement accuracy with a higher percentage of trials overshot compared to their normal weight and overweight peers. In addition, this study found that an increase in target distance and a reduction in target width increased task difficulty coinciding, as expected, with Fitts' law. These findings reinforce the strength of Fitts' law in an original setting as the experimentation took place in a public science gallery and with a larger number of participants than traditionally used. Finally, the modification of tablet orientation was found to highlight differences between BMI categories in terms peak acceleration and overshoot rate.

Further to the existing evidence showing altered motor skill in obese individuals (Smith et al. 2011; Wang et al. 2016), this study sought to investigate whether individuals from normal weight, overweight, and obese groups demonstrate different motor behaviours in a classically used manual aiming task. Surprisingly, there was no significant difference found between BMI categories for overall movement time regardless of tablet orientation, target distance, or target width. This unexpected and interesting finding suggests that obese participants are able to maintain an equivalent level of performance in terms of movement time to their normal weight peers. However, as we looked in greater depth differences between groups emerged, suggesting underlying differences in the control mechanisms in use. Obese participants demonstrated different peak accelerations (Fig. 2), percentage acceleration (Fig. 4), and higher overshoot rate (Fig. 5) than their normal weight and overweight peers during this manual aiming task. Secondly, although non-significant $(p=0.06)$, there was a trend for obese individuals to demonstrate higher peak velocities than their peers for horizontal orientation but lower peak velocities for the vertical orientation. It was unexpected to find between-group differences for peak velocity with the absence of differences

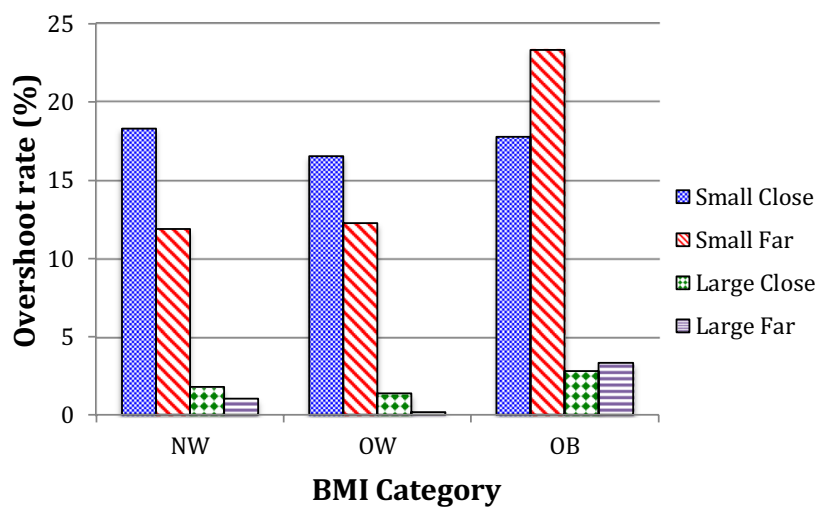

Fig. 5 Percentage of trials which participants' overshot target width shown for normal weight, overweight, and obese participants for all four conditions

for movement time. This would suggest that despite moving faster during the first phase of the movement, obese participants still take the same amount of time to complete the task highlighting a longer deceleration phase in comparison with overweight and normal weight groups. When taken together these findings suggest that obese express difficulties in maintaining the balance between speed and accuracy in their control of movement.

The speed-accuracy trade-off is generally viewed as the consequence of both MT and movement endpoint variance minimization (Meyer et al. 1988; Harris and Wolpert 1998). As we can separate participant's movements into two distinct phases: ballistic and corrective (Elliott et al. 2001), it appears that obese participants' behaviour in each of these phases differs from their normal weight and overweight peers. Although depending on the task constraints in terms of target distance, target width, and tablet orientation, in the first phase, obese participants demonstrate greater and earlier peak acceleration as well as a trend for higher peak velocities. This finding in conjunction with the results for movement time suggests that obese group demonstrate greater impulsivity in the initial ballistic phase of the movement. A parallel can be found in studies examining response inhibition in obese individuals. These studies found that obese individuals demonstrated a more impulsive nature and a poorer response inhibition mechanism compared to normal weighted peers (Lokken et al. 2009; Hendrick et al. 2012; Reyes et al. 2015; Brockmeyer et al. 2016). These higher peak accelerations in the ballistic phase of movement result in greater variance and, therefore, extended deceleration phases to make the required adjustments to maintain accuracy thus maintaining the same overall movement time.

The finding that obese participants demonstrated significantly greater instances of target overshoot, particularly for the most difficult conditions (Fig. 5), can be seen as difficulty in the control of the movement and specifically in the 
corrective phase of the movement. This phase, which deals with adjustment of movement, is an essential component of producing accurate aiming actions. This seems to suggest that obese participants might have difficulty in the utilisation of feedback during movement. This requires them to spend a greater amount of time applying larger corrective adjustments at the end of their movements. Therefore, the lack of movement time differences is a result of a balancing between faster initial movements and greater time spent adjusting at the end of movements. This, in essence, means that the initial movement time gains earned as a result of greater peak acceleration and peak velocities are required to offset costly overshoots and corrective measures at the end of the movement. A study by Heath et al. (1998) found that participants' initial ballistic movements tended to be determined prior to movement initiation and free from online adjustment. It seems that obese participants demonstrated a greater ballistic phase (peak acceleration) that results in higher movement variability and thus requires greater adjustment in the second phase of movement and the associated increase in time decelerating (Harris and Wolpert 1998). When taken all together, these differences in movement kinematics demonstrate that obese participants operate slightly different motor control strategies dependent on the task constraints such as target distance, target width, and orientation of tablet.

Overall, obese participants seem to demonstrate a more varied array of movement characteristics compared to their normal weight and overweight peers when the tablet was in a vertical orientation. This possibly suggests the presence of thresholds with excess mass of the arm adding postural demands in a vertical orientation creating an interference with movement control on goal-directed aiming tasks. However, the lack of incremental group differences from normal weight to overweight to obese participants could perhaps be seen as contrary to the traditional standpoint that excess mass acts as a mechanical constraint. These findings suggest that obese individuals are capable of altering their motor behaviour to preserve motor outcomes, which in turn significantly and acutely affects speed or accuracy.

The findings, that corroborate those traditionally obtained for Fitts' task paradigms, highlight the potential for these approaches to be applied in a more ecological setting. Nevertheless, a number of limitations of this study exist. The less controlled setting and convenience sample limit the generalisability of the results obtained. First, as this study took place in a public exhibition, the reduced controlled setting may have reduced participant's attention. Second, the unbalanced numbers in terms of BMI category, age, and gender also limit the generalisability of the findings, despite the actual distribution of our sample for each BMI category being in line with the population representative sample of the prevalence of overweight and obesity in Ireland. Finally, the authors were also unable to collect additional data on other factors which might play a role in the control of movement such as the number of years participants were overweight or obese, socioeconomic status, comorbidities, or physical activity levels or complementary measures of cognition such as executive function. Despite the limitations discussed, the current study provides a novel approach to the investigation for the potential presence of perceptual motor difficulties in obesity.

The ability to coordinate movement while preserving speed and accuracy underpins all goal-directed aiming tasks. The successful completion of many activities of daily living such as picking up items, brushing one's teeth or buttoning a shirt relies on this ability. As such, difficulties in the preservation of the speed and accuracy balance affect the interaction between individuals and the environment around them. The presence of group differences for obese participants in the quality of movement adds further evidence to the hypothesis that obese disrupts the typical sensory integration process (D'Hondt et al. 2011; Gaul et al. 2016). As participation in physical activity often relies on the ability to coordinate movements quickly and accurately albeit, on a whole body level, problems in this process can result in difficulties participating in such activities. This study adds further weight to the argument for underlying perceptual motor difficulties in obese individuals. However, further research is required to determine whether these problems emerge as a result of the physiological changes when one becomes obese or whether these difficulties exist prior and contribute to becoming obese as a result of a vicious cycle of inactivity.

Acknowledgements The authors gratefully acknowledge the work of Cedric Goulon for his work on development of software used to conduct the experiment.

Funding Ethical approval was granted by Dublin City University's research ethics committee (DCUREC/2011/038). This research was funded by the Government of Ireland Postgraduate Scholarship Scheme 2014 (GOIPG/2014/1516).

\section{Compliance with ethical standards}

Ethical approval All procedures performed in studies involving human participants were in accordance with the ethical standards of the institutional and/or national research committee and with the 1964 Helsinki declaration and its later amendments or comparable ethical standards.

Conflict of interest The authors declare that they have no conflict of interest. 


\section{References}

Alvarez JA, Emory E (2006) Executive function and the frontal lobes: a meta-analytic review. Neuropsychol Rev 16:17-42. https://doi. org/10.1007/s11065-006-9002-x

Benito-Leon J, Mitchell AJ, Hernandez-Gallego J, Bermejo-Pareja F (2013) Obesity and impaired cognitive functioning in the elderly: a population-based cross-sectional study (NEDICES). Eur J Neurol 20:899-906. https://doi.org/10.1111/ene.12083

Beydoun MA, Beydoun HA, Wang Y (2008) Obesity and central obesity as risk factors for incident dementia and its subtypes: a systematic review and meta-analysis. Obes Rev 9:204-218. https:// doi.org/10.1111/j.1467-789X.2008.00473.x

Bove RM, Gerweck AV, Mancuso SM et al (2016) Association between adiposity and cognitive function in young men: hormonal mechanisms. Obesity 24:954-961. https://doi.org/10.1002/oby.21415

Brockmeyer T, Hamze Sinno M, Skunde M et al (2016) Inhibitory control and hedonic response towards food interactively predict success in a weight loss programme for adults with obesity. Obes Facts 9:299-309. https://doi.org/10.1159/000447492

Cournot MC, Marquie JC, Ansiau D, Martinaud C, Fonds H, Ferrieres J, Ruidavets JB (2006) Relation between body mass index and cognitive function in healthy middle-aged men and women. Neurology 67(7):1208-1214

Crichton GE, Elias MF, Buckley JD et al (2012) Metabolic syndrome, cognitive performance, and dementia. J Alzheimers Dis. https:// doi.org/10.3233/JAD-2011-111022

D'Hondt E, Deforche B, De Bourdeaudhuij I et al (2011) Postural balance under normal and altered sensory conditions in normalweight and overweight children. Clin Biomech 26:84-89

Elliott D, Helsen WF, Chua R (2001) A century later: Woodworth's (1899) two-component model of goal-directed aiming. Psychol Bull 127:342-357. https://doi.org/10.1037/0033-2909.127.3.342

Etnier JL, Chang Y-K (2009) The effect of physical activity on executive function: a brief commentary on definitions, measurement issues, and the current state of the literature. J Sport Exerc Psychol 31:469-483. https://doi.org/10.1123/jsep.31.4.469

Fitts PM (1954) The information capacity of the human motor system in controlling the amplitude of movement. J Exp Psychol 47(6):381-391

Fitzpatrick AL, Kuller LH, Lopez OL, Diehr P, O'Meara ES, Longstreth WT, Luchsinger JA (2009) Midlife and late-life obesity and the risk of dementia. Arch Neurol 66(3):336-342

Gaul D, Mat A, O'Shea D, Issartel J (2016) impaired visual motor coordination in obese adults. J Obes 2016:1-8. https://doi. org/10.1155/2016/6178575

Gunstad J, Lhotsky A, Wendell CR, Ferrucci L, Zonderman AB (2010) Longitudinal examination of obesity and cognitive function: results from the Baltimore longitudinal study of aging. Neuroepidemiology 34(4):222-229

Gustafson D (2008) A life course of adiposity and dementia. Eur J Pharmacol 585(1):163-175

Harris CM, Wolpert DM (1998) Signal-dependent noise determines motor planning. Nature 394:780-784. https://doi. org/10.1038/29528

Heath M, Hodges NJ, Chua R, Elliott D (1998) On-line control of rapid aiming movements: unexpected target perturbations and movement kinematics. Can J Exp Psychol/Revue canadienne de psychologie expérimentale 52(4):163-173

Hendrick OM, Luo X, Zhang S, Li CR (2012) Saliency processing and obesity: a preliminary imaging study of the stop signal task. Obesity 20:1796-1802. https://doi.org/10.1038/oby.2011.180

Kirby A, Edwards L, Sugden D (2011) Emerging adulthood in developmental co-ordination disorder: parent and young adult perspectives. Res Dev Disabil 32:1351-1360. https://doi. org/10.1016/j.ridd.2011.01.041

Kivipelto M, Ngandu T, Fratiglioni L et al (2005) Obesity and vascular risk factors at midlife and the risk of dementia and Alzheimer disease. Arch Neurol 62:1156-1560

Knecht S, Ellger T, Levine JA (2008) Obesity in neurobiology. Prog Neurobiol 84:85-103. https://doi.org/10.1016/j.pneur obio.2007.09.003

Kramer AF, Humphrey DG, Larish JF, Logan GD (1994) Aging and inhibition: beyond a unitary view of inhibitory processing in attention. Psychol Aging 9:491-512. https://doi. org/10.1037/0882-7974.9.4.491

Lee I-M, Shiroma EJ, Lobelo F et al (2012) Effect of physical inactivity on major non-communicable diseases worldwide: an analysis of burden of disease and life expectancy. Lancet 380:219-229. https ://doi.org/10.1016/S0140-6736(12)61031-9

Liang J, Matheson BE, Kaye WH, Boutelle KN (2014) Neurocognitive correlates of obesity and obesity-related behaviors in children and adolescents. Int J Obes 38:494-506. https://doi.org/10.1038/ ijo.2013.142

Lokken KL, Boeka AG, Austin HM et al (2009) Evidence of executive dysfunction in extremely obese adolescents: a pilot study. Surg Obes Relat Dis 5:547-552. https://doi.org/10.1016/j.soard .2009 .05 .008

Meyer DE, Abrams RA, Kornblum S et al (1988) Optimality in human motor performance: ideal control of rapid aimed movements. Psychol Rev 95:340-370. https://doi. org/10.1037/0033-295X.95.3.340

Miller AA, Spencer SJ (2014) Obesity and neuroinflammation: a pathway to cognitive impairment. Brain Behav Immun 42:10-21. https ://doi.org/10.1016/j.bbi.2014.04.001

Missenard O, Fernandez L (2011) Moving faster while preserving accuracy. Neuroscience 197:233-241. https://doi.org/10.1016/j. neuroscience.2011.09.020

Plamondon R, Alimi AM (1997) Speed/accuracy trade-offs in targetdirected movements. Behav Brain Sci 20(02):279-303

Prickett C, Brennan L, Stolwyk R (2014) Examining the relationship between obesity and cognitive function: a systematic literature review. Obes Res Clin Pract 9:1-21. https://doi.org/10.1016/j. orcp.2014.05.001

Reyes S, Peirano P, Peigneux P et al (2015) Inhibitory control in otherwise healthy overweight 10-year-old children. Int J Obes 39:1230 1235. https://doi.org/10.1038/ijo.2015.49

Rosmond R, Bjorntorp P (2000) Quality of life, overweight, and body fat distribution in middle-aged men. Behav Med 26:90-94. https ://doi.org/10.1080/08964280009595757

Scarpina F, Migliorati D, Marzullo P et al (2016) Altered multisensory temporal integration in obesity. Sci Rep 6:1-7. https://doi. org/10.1038/srep28382

Smith E, Hay P, Campbell L, Trollor JN (2011) A review of the association between obesity and cognitive function across the lifespan: implications for novel approaches to prevention and treatment. Obes Rev 12:740-755. https://doi.org/10.1111/j.1467789X.2011.00920.x

Wan X, Spence C, Mu B et al (2014) Assessing the benefits of multisensory audiotactile stimulation for overweight individuals. Exp Brain Res 232:1085-1093. https://doi.org/10.1007/s0022 1-013-3792-x

Wang C, Chan JSY, Ren L, Yan JH (2016) Obesity reduces cognitive and motor functions across the lifespan. Neural Plast 2016:1-13. https://doi.org/10.1155/2016/2473081

Whitmer RA, Gunderson EP, Barrett-Connor E et al (2005) Obesity in middle age and future risk of dementia: a 27 year longitudinal population based study. BMJ 330(7504):1360. https://doi. org/10.1136/bmj.38446.466238.E0 
WHO (2000) Obesity: preventing and managing the global epidemic. Report of a WHO consultation, vol 894. World Health Organization, Geneva. pp 1-253 (ISBN 9241208945 )
World Health Organization (2014) Factsheet: obesity and overweight. World Health Organization, Geneva 